\title{
Important feature of calculating bridges under seismic action
}

\author{
Lyubov Smirnova ${ }^{1 *}$, Alexander Uzdin $^{2}$, Natalia Polorotova ${ }^{2}$, and Maxim Freze $^{3}$ \\ ${ }^{1}$ JSC Research Center of Construction, 2-nd Institutskaya st., 6, Moscow, 109428, Russia \\ 2 Emperor Alexander I St. Petersburg State Transport University, Moskovsky pr., 9, Saint- \\ Petersburg, 190031, Russia \\ ${ }^{3}$ Transmost JSC, Podyezdnoy per., 1, Saint-Petersburg, 190013, Russia
}

\begin{abstract}
The purpose of the research is to show the main features of calculating bridge taking into account the inhomogeneous acceleration field along the structure length. The bridge is considered to be a linear structure with point bearings on the soil base. For long bridges it is typical, that their bearings are located in different seismogeological conditions. This result in inhomogeneity of the acceleration field under the piers and non-synchronous pier excitations. The motion equations of the system under consideration are constructed and their decomposition into vibration modes is performed without the account of external and internal damping in the system and with the account of it. Based on the proposed decomposition, formulas for determining seismic loads taking into account various seismicity under piers are obtained. The result obtained show that the peculiarities considered can be easily taken into account in existing software packages. As an example, the authors analyzed the results of calculating a four-span beam railway bridge. In calculating it was taken into account that the first and second piers are located on sandstone, and the rest of them are on water-saturated loose sand. The analysis showed that the account of the non-synchronous support point excitation of the extended system reduces inertial seismic loads on its elements significantly.
\end{abstract}

\section{Introduction}

Bridges have a number of fundamental features that significantly distinguish their work during earthquakes from civil and industrial construction. Professor G.N. Kartsivadze made a great contribution to studies of these features $[1,2]$. Thanks to his work in the former USSR from the mid-70s of the last century the issues of seismic bridge resistance were singled out in a separate section of the general theory of earthquake engineering. Over the past 40 years, serious research has been devoted to the bridge seismic resistance. The issues of evaluating seismic loads on bridges are considered in the monograph by G.S. Shestoperov [3]. A modern analysis of the bridge seismic stability is given in the monograph [4]. One of the important specific features of bridges is their length. In many cases such concept as the construction site seismicity loses its meaning for the bridge.

*Corresponding author: lyubovsmirnova80@ gmail.com 
Usually, river bed piers are located in poor soil conditions, which may require an increase of the design seismicity of the construction site per unit as compared with the area seismicity. At the same time, bank piers can be placed on a bedrock, which reduces the design seismicity by a unit. For longitudinal calculation of simple girder bridges G.N. Karzivadze substantiated the ability to consider each pier independently [2]. He carried out similar studies for transverse vibrations of bridges with a regular structure, in which stiffnesses of adjacent piers differ slightly.

At present, bridges, especially road bridges, have become noticeably more diverse: continuous, frame, cable-stayed bridges, viaducts over deep canyons and others are widely used. The use of seismic isolating bearings becomes the basic solution of seismic protection $[4,5]$. The calculation of such bridges should be done using a unified structural system taking into account an inhomogeneous field of seismic excitation along the bridge length. In this case, the bridge is considered as an extended linear structure with a point bearing on the base. Lately, a lot of theoretical research has been devoted to calculating extended structures with point bearing on the base. In Russia, these issues are considered in the monograph by Nazarov Yu.P. [6], publications by Poznyak E.V. [7], Savvas P. et al. [8], Uzdin A.M. \& Dmitrovskaya L.N. [9], as well as in the abovementioned monograph [4]. Abroad, fundamental research of the systems under consideration was carried out by A. Der-Kiureghian [10,11]. However, the practical application of the theory of calculating extended multi-support structures has caused certain difficulties so far. A description of these difficulties and ways of overcoming them are discussed below on the example of calculating a specific bridge.

The paper considers the transverse calculation of a four-span beam railway bridge i.e. displacements from the drawing plane are considered. The bridge is located in a seismically dangerous area with seismicity 8 on the MSK scale. Piers 0 and 1 are located on sandstone, which belongs to the first category soils and allows one to reduce the design seismicity by unit. The remaining piers are located on water-saturated loose sand belonging to the third category soils, which requires an increase in background seismicity by the unit. Thus, the seismicity of the construction site for the first two piers is 7 degree on the MSK scale and for the third, fourth and fifth piers are 9 degree on the MSK scale.

A schematic drawing of the bridge is shown in Figure 1. The design scheme of the bridge is shown in Figure 2. To illustrate the features of calculating the bridge, taking into account the inhomogeneous acceleration field along its length, a system with concentrated masses with 12 degrees of freedom is considered. Moreover, the system has 5 bearing nodes characterizing seismic input.

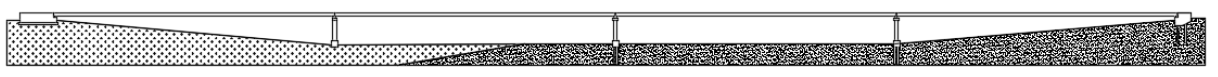

Fig. 1. Schematic drawing of the bridge

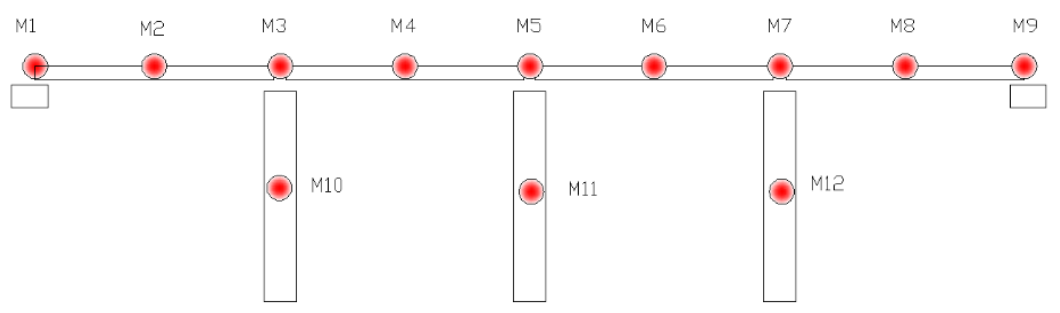

Fig.2. Design scheme of the bridge with concentrated masses 


\section{Materials and Methods}

Let us write the initial oscillation equation for a heterogeneous excitation field along the structure length. According to [21] it has the form

$$
\mathbf{M} \ddot{\mathbf{Y}}+\left(\mathbf{B}_{\mathbf{I}}+\mathbf{B}_{\mathbf{E}}\right) \dot{\mathbf{Y}}+\mathbf{R Y}=\left(\mathbf{M R}^{-1} \mathbf{C}-\mathbf{m}_{1}\right) \ddot{\mathbf{Y}}_{\mathbf{0}}+\left(\mathbf{B}_{\mathbf{I}}+\mathbf{B}_{\mathbf{E}}\right) \mathbf{R}^{-1} \mathbf{C} \dot{\mathbf{Y}}_{\mathbf{0}}-\boldsymbol{\beta}_{\mathrm{I}} \dot{\mathbf{Y}}_{\mathbf{0}}-\boldsymbol{\beta}_{\mathbf{E}} \dot{\mathbf{Y}}_{\mathbf{0}}
$$

Here $\mathbf{M}$ is the inertia matrix; $\mathbf{Y}$ is the displacement of the system nodes relative to the position caused by the displacements of the bearing nodes of the weightless system; $\mathbf{B}_{\mathrm{I}}, \mathbf{B}_{\mathrm{E}}$ are matrixes of external and internal damping of the system; index I refers to internal nodes, and $\mathrm{E}$ refers to external nodes; $\mathbf{R}$ is the system stiffness matrix; $\mathbf{C}$ is the reaction matrix in the direction of the generalized coordinates from the displacement of the bearing nodes; $\mathbf{m}_{1}$ is the inertia matrix corresponding to the nodes at the boundary of the computational domain of the base. This equation is somewhat different from those traditionally used in books $[4,12]$ and Russian Seismic Building Design Code SP 14.13330.2018.

First, in the equation right-hand side, the matrix of inertia before the vector $\mathbf{Y}_{0}$ is multiplied by the matrix $\mathbf{C}$ and supplemented by a member $\mathbf{m}_{1}$. The presence of this member is well known in structure dynamics [5]. In Russian literature on earthquake engineering the presence of this member was indicated V.A. Semenov and A.G. Tyapin [13,14]. In finite element calculations, when the inertia matrix $\mathrm{M}$ is determined by basic functions, such member inevitably appears. To simplify the problem, we consider a computational model with concentrated masses, i.e. we use the diagonal mass matrix.

Secondly, external and internal damping are separated in the equation. In this case, the presence of external damping leads to the appearance of an additional member on the right side of the oscillation equation. In this design model, there is no external damping. As shown in [14], $\mathbf{B}_{\mathbf{I}} \mathbf{R}^{-1} \mathbf{C}-\boldsymbol{\beta}_{\mathbf{I}}=0$, which also simplifies the equation.

Thirdly, pier excitations are accepted to be heterogeneous. It is determined by the vector $\ddot{\mathbf{Y}}_{\mathbf{0}}$. If all the supporting points are excited to one law and therefore, have one response spectrum, but excitation amplitudes under each pier are characterized by their value determined by the vector $\hat{\mathbf{I}}$, then $\ddot{\mathbf{Y}}_{\mathbf{0}}=\hat{\mathbf{I}} \ddot{y}_{0}$.

As a result, the resolving equation system gets the form

$$
\mathbf{M Y}+\mathbf{B}_{\mathbf{I}} \dot{\mathbf{Y}}+\mathbf{R Y}=\mathbf{M} \mathbf{R}^{-1} \mathbf{C} \ddot{\mathbf{I}}_{0}
$$

This equation coincides with the classical one if the vector $\mathbf{V}_{\mathrm{p}}$ of the action projections on the generalized coordinates directions is taken equal to $\mathbf{R}^{-1} \mathbf{C} \hat{\mathbf{I}}$

Note, that the proposed form (2) allows one to the calculate of a bridge when only one support is excited. To do this, in the vector $\hat{\mathbf{I}}$ one must set the value corresponding to the considered pier equal to 1 , and set the remaining elements of the vector to zero. This technique allows one to set its own impact and its response spectrum (dynamic curve) under each pier.

Further we expand equation (2) according to the oscillation modes of a non-damped system, i.e., we present solution (2) in the form

$$
\mathbf{Y}=\mathbf{X} \boldsymbol{\Xi}
$$

Here $\mathbf{X}$ is the matrix of eigenvectors of the matrix $\mathbf{M}^{-1} \mathbf{R}$, i.e. eigenvector matrix of a nondamped system; $\boldsymbol{\Xi}=\left\{\xi_{1}, \xi_{2}, \ldots \xi_{\mathrm{n}}\right\}$ is the vector of principal coordinates.

The matrix $\mathbf{X}$ satisfies the well-known condition 


$$
\mathbf{M}^{-1} \mathbf{R X}=\mathbf{X} \Lambda=\mathrm{XK}^{2}
$$

Where $\Lambda=\mathbf{K}^{2}$ is the eigenvalues matrix of the matrix $\mathbf{M}^{-1} \mathbf{R}, \mathbf{K}=\left\lceil\mathrm{k}_{1}, \mathrm{k}_{2}, \ldots \mathrm{k}_{\mathrm{n}}\right\rfloor$ is the diagonal matrix of the eigenfrequencies.

Substituting (3) into (2) gives $\mathbf{M X \ddot { \Xi }}+\mathbf{B}_{\mathbf{I}} \mathbf{X} \dot{\boldsymbol{\Xi}}+\mathbf{R Y}=-\mathbf{M} \mathbf{R}^{-1} \mathbf{C} \hat{\mathbf{I}} \ddot{y}_{0}$.

After multiplying this equation by $\mathbf{X}^{-1} \mathbf{M}^{-1}$ and taking into account (4), we obtain

$$
\ddot{\Xi}+\mathbf{X}^{-1} \mathbf{M}^{-1} \mathbf{B}_{\mathbf{I}} \mathbf{X} \dot{\boldsymbol{\Xi}}+\mathbf{K}^{2} \boldsymbol{\Xi}=-\mathbf{X}^{-1} \mathbf{R}^{-1} \mathbf{C} \hat{\mathbf{I}} \ddot{y}_{0}
$$

In the general case, the matrix $\mathbf{X}^{-1} \mathbf{M}^{-1} \mathbf{B}_{\mathbf{I}} \mathbf{X}$ is not diagonal, and the system is not divided into independent equations. In the particular case when the matrices $\mathbf{M}^{-1} \mathbf{B}$ and $\mathbf{M}^{-1} \mathbf{R}$ have the same system of eigenvectors, the equation system is divided [15]. In this case, damping is called proportional. Adopting

$$
\mathbf{X}^{-1} \mathbf{M}^{-1} \mathbf{B}_{\mathbf{I}} \mathbf{X}=\chi_{=}=\left\lceil\chi_{1}, \chi_{2}, \ldots \chi_{\mathrm{n}}\right\rfloor,
$$

we get a divided equation system

$$
\ddot{\boldsymbol{\Xi}}+\chi \dot{\boldsymbol{\Xi}}+\mathbf{K}^{2} \boldsymbol{\Xi}=-\mathbf{X}^{-1} \mathbf{R}^{-1} \mathbf{C} \hat{\mathbf{I}}_{0}
$$

As a result, the system decomposes into independent equations of the form

$$
\ddot{\xi}_{j}+\chi_{j} \dot{\xi}_{j}+k_{j}^{2} \xi_{j}=-d_{j} \ddot{y}_{0}
$$

where $\mathrm{d}_{\mathrm{j}}$ is a member of vector $\mathbf{X}^{-1} \mathbf{R}^{-1} \mathbf{C} \hat{\mathbf{I}}$.

It is commonly accepted that $\chi_{\mathrm{j}}=\gamma_{\mathrm{j}} \cdot \mathrm{k}_{\mathrm{j}}$.

The quantity $\gamma_{j}$ has the meaning of the coefficient of inelastic resistance. We refer to these coefficients as the modal damping, and the matrix $\boldsymbol{\Gamma}=\left\lceil\gamma_{1}, \gamma_{2}, \ldots \gamma_{\mathrm{n}}\right\rfloor$ is called damping spectrum.

To calculate complex systems, instead of inverting the matrix of eigenvectors, one can use its orthogonality with weight

$$
\mathbf{X}^{T} \mathbf{M X}=\mathbf{N}
$$

where $\mathbf{N}=\left\lceil v_{1}, v_{2}, \ldots v_{n}\right\rfloor$ is the diagonal matrix.

For the diagonal matrix of masses $v_{j}=\sum_{i=1}^{n} x_{i j}^{2} m_{i}$.

Seismic forces $\mathrm{s}_{\mathrm{ij}}$ are calculated according to vibration modes using the well-known formula

$$
s_{i j}=m_{i} A K_{1} g \beta\left(T_{j}\right) K_{\Psi}\left(\gamma_{j}\right) \eta_{i j}
$$

where $m_{i}$ is the $i-t h$ system mass; $A$ is the value of the base acceleration in fractions of the gravity acceleration $\mathrm{g} ; \mathrm{K}_{1}$ is coefficient which takes into account permissible damage to buildings and structures; $\beta\left(T_{j}\right)$ is the value of the response spectrum; $K_{\Psi}\left(\gamma_{j}\right)$ is the coefficient which takes into account the ability of buildings and structures to dissipate energy; $\eta_{\mathrm{ij}}$ is an analogue of the participant mode factor.

For the mode participant factor $\eta_{\mathrm{ij}}$, the well-known formula is used

$$
\eta_{i j}=\frac{x_{i j} \sum_{k=1}^{n} x_{k j} m_{k} V_{p}^{(k)}}{v_{k}}
$$


In contrast to the traditional formula for the mode participant factor, here $V_{p}^{(k)}$ is an element of the vector $\mathbf{R}^{-1} \mathbf{C} \hat{\mathbf{I}}$.

$x_{i j}$ is the offsets in the $\mathrm{i}$-th form in the direction of the generalized coordinate; $m_{k}$ is the inertial characteristics at the nodal point $\mathrm{k}$.

If the acceleration field along the length of the bridge is one-valued and determined, then we can restrict ourselves to calculating system loads caused by seismic forces and add them to the loads from the support displacements. However, in fact, the situation is usually different. The pier excitations are independent random functions. In this case, it is necessary to carry out calculations for each pier excitations. This will result in many seismic force matrices the number of which is equal to the number of piers. The matrix of seismic forces from the excitation of the k-th pier will have the form $\mathbf{S}_{k}=\left\|S_{i j}^{(k)}\right\|$. These forces will cause strains, displacements and other factors the system behavior.

Let us denote the value of the $\mathrm{r}$ - $t$ f factor in mode $\mathrm{j}$ from the excitation of the $\mathrm{k}$-th pier by $\Phi_{r j}^{(k)}$, and the value of this factor from the displacement of the k-th pier $\phi_{r}^{(k)}$. Then the value of the factor is determined by the following sum

$$
F_{r}^{(\text {calc })}=\left[\sum_{k=1}^{n}\left\{\left(\phi_{r}^{(k)}\right)^{2}+\sum_{j=1}^{N f} \sum_{p=1}^{N f} \Phi_{r j}^{(k)} \Phi_{r p}^{(k)} \varepsilon_{j p}\right\}\right]^{1 / 2},
$$

where $\varepsilon_{\mathrm{jp}}$ is the correlation coefficient of the $\mathrm{j}$-th and $\mathrm{p}$-th vibration modes. To calculate the correlation coefficient, the formulas of Newmark [16] and A. Der-Kiureghian [10,11,12] can be used. In Russia the formula of correlation coefficient was proposed by A.A. Petrov (Petrov A.A., Bazilevskij S.V. Uchet vzaimnoj korrelyacii mezhdu obobshchennymi koordinatami pri opredelenii sejsmicheskih nagruzok. - Ref.inf. "Sejsmostojkoe stroitel'stvo (otechestvennyj $i$ zarubezhnyj opyt)», seriya XIV, CINIS, M., 1978, vyp.5, p.23-28) and widely used in engineering practice $[17,18]$.

When only the correlation between the support excitation is taken into account, expression (12) takes the form

$$
F_{r}^{(c a l c)}=\left[\sum_{k=1}^{n} \sum_{s=1}^{n}\left\{\left(\phi_{r}^{(k)} \phi_{r}^{(s)}\right)+\sum_{j=1}^{N f}\left(\Phi_{r j}^{(k)} \Phi_{r j}^{(s)}\right)+\phi_{r}^{(k)} \sum_{j=1}^{N f}\left(\Phi_{r j}^{(s)}\right)\right\} \rho_{k s}\right]^{1 / 2},
$$

where $\rho_{\mathrm{ks}}$ is the correlation coefficient between excitations of the k-th and s-th pier. It is believed that on non-rocky soils, correlation occurs when the distance between the bearing points is less than $60 \mathrm{~m}$ [19]. In other cases, to estimate the value $\rho_{\mathrm{ks}}$ formulas based on the hypothesis of a frozen wave are available [20].

\section{Results}

\subsection{RSM calculation of a bridge with a homogeneous acceleration field}

For a homogeneous acceleration field, one calculation of seismic loads was performed. According to Russian Seismic Building Design Code SP 14.13330.2018 do not provide setting different accelerations under piers, a synchronous pier excitations have been accepted in the reserve, based on the design seismicity 9 on the MSK scale. To take account of inhomogeneous damping, a method of distributing energy losses by vibration modes in proportion to the mode energy was used. As is known, this approach result in a proportional 
damping matrix. The design seismic loads are calculated using the normative formula (formula (12)). The calculation results are shown in table 1.

In table 1 , the load values of dominating among the loads in the vibration mode (in the matrix column) are mark out in a brighter type, and the cells of the table, in which are the loads that determine the total load on the corresponding mass (in the matrix row), are mark out in beige and yellow colors.

Table 1. Seismic mode loads for identical pier excitations

\begin{tabular}{|c|c|c|c|c|c|c|c|c|c|c|c|c|c|}
\hline \multirow{2}{*}{$\begin{array}{c}\text { № } \\
\text { mass }\end{array}$} & \multicolumn{12}{|c|}{ Mode loads } & \multirow{2}{*}{$\begin{array}{l}\text { Sum } \\
\text { mary }\end{array}$} \\
\hline & 1 & 2 & 3 & 4 & 5 & 6 & 7 & 8 & 9 & 10 & 11 & 12 & \\
\hline 2 & -0.0 & 0.3 & 0 & -116.3 & 0 & -394.6 & -13.6 & -60.6 & 1744 & -266.4 & 1509 & -43.2 & 2359 \\
\hline 4 & 0.1 & -0.3 & 0 & -1.6 & -0.1 & -863.0 & -10.9 & 39.2 & 3768 & 374.3 & -1065 & -31.4 & 4027 \\
\hline 5 & -36.2 & -263.2 & -0.6 & 0.8 & 1.1 & 2153 & 1.9 & -228.5 & 1385 & -21.4 & -310.7 & 0.9 & 2603 \\
\hline 6 & -0.1 & -0.4 & 0.2 & 0 & -3.5 & -844.2 & 13.5 & 4.8 & 3454 & -569.2 & 105.7 & 46.2 & 3603 \\
\hline 9 & 0 & 0 & 0.3 & 0 & 2632 & -56.8 & -5.9 & -5.9 & 43.7 & 49.3 & 45.0 & 1.2 & 1634 \\
\hline 10 & -52.4 & 600.3 & 1.5 & 31.7 & 0 & 677.6 & 72.0 & 72.0 & 433.7 & 4.0 & 92.7 & -7.4 & 1013 \\
\hline 11 & 66.7 & 485.3 & 1.1 & 0.3 & 0.5 & 958.4 & -101.1 & -101.1 & 594.5 & -9.0 & -134.7 & 0.4 & 1241 \\
\hline 12 & -0.6 & -14.0 & 570.3 & 0 & 22.4 & 710.3 & 67.3 & 67.3 & 405.6 & 15.7 & 106.6 & 7.5 & 1006 \\
\hline
\end{tabular}

\subsection{RSM calculation of the bridge taking into account the heterogeneity of the acceleration field}

To illustrate the significance of the effect of heterogeneity of the acceleration field along the length of the bridge, we restrict ourselves to estimating the seismic load using formulas (10). Note that the system under consideration is statically determinable and no stresses caused by mutual pier displacements arise in it. Since the system has 5 bearing nodes, 5 matrices of seismic forces from each support excitation were obtained. For these calculations the PGA is assumed to be $1 \mathrm{~m} / \mathrm{s}^{2}$ for the first two piers, which corresponds to the construction site seismicity equal to 7 degree on the MSK scale, and the response spectrum (dynamic curve) is adopted for the rock base. At the same time, for the next 4 piers, PGA $=4 \mathrm{~m} / \mathrm{s}^{2}$, which corresponds to the construction site seismicity equal to the 9 degree on the MSK scale, and the response spectrum (dynamic curve) is accepted for a weak base. As an example, tables 2 , 3 and 4 show the matrices of seismic forces according to the oscillation modes from different excitations under piers. Seismic forces caused by synchronous excitations with different amplitudes under piers are shown in table 2. Seismic forces caused by nonsynchronous excitations of the second and third piers are shown in tables 3 and 4 . The conclusive calculation results are given in table 5 .

Table 2. Seismic mode loads for synchronous pier excitations with different amplitudes under piers

\begin{tabular}{|c|c|c|c|c|c|c|c|c|c|c|c|c|c|}
\hline \multirow{2}{*}{$\begin{array}{c}\text { № } \\
\text { mass }\end{array}$} & \multicolumn{12}{|c|}{ Mode loads } & \multirow{2}{*}{ Summary } \\
\hline & 1 & 2 & 3 & 4 & 5 & 6 & 7 & 8 & 9 & 10 & 11 & 12 & \\
\hline 1 & -0.4 & 0.6 & 0 & 4.7 & 0 & 44.7 & -27.7 & -6.6 & -38.7 & 2.2 & -1.8 & 29.7 & 72.2 \\
\hline 2 & 0.1 & -0.1 & 0 & 0.2 & 0 & 304.4 & -213.1 & -59.6 & -1278 & 50.8 & -46.9 & 869.1 & 1592 \\
\hline 3 & $\begin{array}{l}-64.3 \\
\end{array}$ & 106.4 & 0.5 & \begin{tabular}{|c|}
-0.1 \\
\end{tabular} & 0 & -1175 & 708.1 & 160.0 & -741.5 & -1.8 & -6.6 & 344.5 & 1610 \\
\hline 4 & -0.2 & 0.1 & 0 & 0 & 0 & 665.8 & -171.5 & 38.5 & 2762 & -71.4 & 33.06 & 630.4 & 2917 \\
\hline 5 & 82.0 & 86.1 & 0.3 & 0 & 0 & -1661 & 29.1 & -224.7 & -1015 & 4.07 & 9.6 & -17.4 & 1964 \\
\hline 6 & 0.1 & 0.1 & -0.1 & 0 & 0 & 651.2 & 211.6 & 4.7 & -2533 & 108.6 & -3.3 & -928.4 & 2785 \\
\hline 7 & -0.8 & -2.5 & 170.7 & 0 & 0 & -1233 & -695.8 & 149.6 & -694.1 & -7.2 & -7.7 & -349.8 & 1631 \\
\hline 8 & 0 & 0 & 0.2 & 0 & 0 & 246.8 & 163.1 & -43.8 & -802.5 & -173.8 & -28.0 & -568.5 & 1043 \\
\hline 9 & 0 & 0 & -0.2 & 0 & 0.5 & 43.8 & 25.3 & -5.8 & -32.0 & -9.4 & -1.4 & -25.02 & 65.83 \\
\hline 10 & 118.5 & -196.3 & -0.8 & -0.1 & 0 & -522.7 & 314.2 & 70.8 & -318.0 & -0.8 & -2.9 & 148.6 & 743.5 \\
\hline 11 & 151.1 & -158.7 & -0.7 & 0 & 0 & -739.3 & 12.4 & -99.5 & -435.9 & 1.72 & 4.2 & -7.2 & 891.5 \\
\hline 12 & 1.4 & 4.6 & -315.0 & 0 & 0 & -548.0 & -308.5 & 66.2 & -298.1 & -3.0 & -3.3 & -150.7 & 781.4 \\
\hline
\end{tabular}

Table 3. Mode seismic loads with excitation of pier No. 2

\begin{tabular}{|c|c|c|c|c|c|c|c|c|c|c|c|c|c|}
\hline \multirow{2}{*}{ № mass } & \multicolumn{12}{|c|}{ Mode loads } & \multirow{2}{*}{ Summary } \\
\hline & 1 & 2 & 3 & 4 & 5 & 6 & 7 & 8 & 9 & 10 & 11 & 12 & \\
\hline 1 & 0.1 & 0.2 & 0 & -6. & 0 & 4.1 & 10 & 6.6 & -3.7 & 0.2 & -5.6 & -8.67 & 17.9 \\
\hline 2 & 0 & -0.1 & 0 & 0.3 & 0 & 27.9 & 76.9 & 59.7 & -123.6 & 3.8 & -139.6 & -253.4 & 330.5 \\
\hline
\end{tabular}




\begin{tabular}{|l|r|r|r|r|r|r|r|r|r|r|r|r|r|}
\hline $\mathbf{3}$ & 16.2 & 25.7 & 0 & -0.2 & 0 & -107.4 & -255.8 & -160.5 & -71.7 & -0.2 & -19.7 & -100.4 & 345.4 \\
\hline $\mathbf{4}$ & 0.1 & 0.1 & 0 & 0 & 0 & 60.9 & 61.9 & -38.7 & -267.2 & -5.3 & 98.5 & -183.8 & 351.9 \\
\hline $\mathbf{5}$ & -20.6 & 20.8 & 0 & 0 & 0 & -151.9 & -10.5 & 225.3 & -98.2 & 0.3 & 28.7 & 5.1 & 292.1 \\
\hline $\mathbf{6}$ & -0.1 & 0.1 & 0 & 0 & 0 & 59.6 & -76.5 & -4.7 & -244.9 & 8.1 & -9.8 & 270.7 & 377.9 \\
\hline $\mathbf{7}$ & 0.2 & -0.6 & 0.4 & 0 & 0 & -112.8 & 251.4 & -150.0 & -67.1 & -0.5 & -22.9 & 101.9 & 337.4 \\
\hline $\mathbf{8}$ & 0 & 0 & 0 & 0 & 0 & 22.6 & -58.9 & 43.9 & -77.6 & -12.9 & -83.5 & 165.7 & 215.8 \\
\hline $\mathbf{9}$ & 0 & 0 & 0 & 0 & 0 & 4.0 & -9.2 & 5.8 & -3.1 & -0.7 & -4.2 & 7.3 & 14.6 \\
\hline $\mathbf{1 0}$ & -29.8 & -47.5 & 0 & -0.1 & 0 & -47.8 & -113.5 & -70.9 & -30.8 & -0.1 & -8.6 & -43.3 & 162.0 \\
\hline $\mathbf{1 1}$ & 38.0 & -38.4 & 0 & 0 & 0 & -67.6 & -4.5 & 998 & -42.2 & 0.1 & 12.5 & 2.1 & 139.3 \\
\hline $\mathbf{1 2}$ & -0.4 & 1.1 & -0.8 & 0 & 0 & -50.1 & 111.5 & -66.4 & -28.8 & -0.2 & -9.9 & 43.9 & 148.9 \\
\hline
\end{tabular}

Table 4. Mode seismic loads with excitation of pier No. 3

\begin{tabular}{|c|c|c|c|c|c|c|c|c|c|c|c|c|c|}
\hline \multirow{2}{*}{$\begin{array}{c}\text { № } \\
\text { mass }\end{array}$} & \multicolumn{12}{|c|}{ Mode loads } & \multirow{2}{*}{ Summary } \\
\hline & 1 & 2 & 3 & 4 & 5 & 6 & 7 & 8 & 9 & 10 & 11 & 12 & \\
\hline 1 & -0.5 & 0.5 & 0 & 0 & 0 & 23.5 & 1.6 & -37.8 & -20.5 & -1.4 & 33.1 & 2.0 & 59.202 \\
\hline 2 & 0.1 & -0.1 & 0 & 0 & 0 & 160.3 & 11.9 & -340.1 & -678.5 & -32.1 & 830.9 & 58.6 & 1139 \\
\hline 3 & -80.4 & 81.0 & 0 & 0 & 0 & -618.4 & -39.6 & 913.8 & -393.5 & 1.2 & 116.9 & 23.2 & 1184 \\
\hline 4 & -0.2 & 0.1 & 0 & 0 & 0 & 350.5 & 9.6 & 220.1 & -1466 & 45.0 & -586.3 & 42.5 & 1633 \\
\hline \begin{tabular}{|l}
5 \\
\end{tabular} & 102.4 & 65.5 & 0 & 0 & 0 & -874.5 & -1.6 & -1283 & -538.9 & -2.6 & -171.1 & -1.2 & 1657 \\
\hline 6 & 0.2 & 0.1 & 0 & 0 & 0 & 342.9 & -11.8 & 27.0 & -1344 & -68.5 & 58.2 & -62.6 & 1392 \\
\hline 7 & -0.9 & -1.9 & 3.1 & 0 & 0 & -649.4 & 38.9 & 854.4 & -368.4 & 4.5 & 136.3 & -23.6 & 1144 \\
\hline 8 & 0 & 0 & 0 & 0 & 0 & 129.9 & -9.1 & -250.2 & -425.9 & 109.5 & 497.0 & -38.32 & 722.2 \\
\hline 9 & 0 & 0 & 0 & 0 & \begin{tabular}{l|}
-0.2 \\
\end{tabular} & 23.1 & -1.4 & -32.9 & -16.9 & 5.9 & 24.8 & -1.7 & 50.6 \\
\hline 10 & 148.1 & -149.5 & 0 & 0 & 0 & -275.2 & -17.6 & 404.2 & -168.8 & 0.5 & 51.0 & 10.0 & 561.1 \\
\hline 11 & -188.7 & -120.8 & -0.1 & 0 & 0 & -389.2 & -0.7 & -568.1 & -231.3 & -1.1 & -74.2 & -0.5 & 763.8 \\
\hline 12 & 1.7 & 3.5 & -5.7 & 0 & 0 & -288.5 & 17.3 & 377.9 & -158.2 & 1.9 & 58.7 & -10.2 & 504.9 \\
\hline
\end{tabular}

The calculation data as compared with the calculation data for the synchronous pier excitations are shown in table 5.

Table 5. Seismic load calculation results

\begin{tabular}{|c|c|c|c|c|c|c|c|c|}
\hline \multirow{3}{*}{ № mass } & \multicolumn{8}{|c|}{ Modal forces and design loads } \\
\hline & \multicolumn{2}{|c|}{$\begin{array}{c}\text { For synchronous pier } \\
\text { excitations }\end{array}$} & \multicolumn{6}{|c|}{ For nonsynchronous pier excitations } \\
\hline & Identical & Different & $\mathrm{N}_{1}$ & $\mathrm{~N}_{2}$ & $\mathrm{~N}_{3}$ & $\mathrm{~N}_{4}$ & $\mathrm{~N}_{5}$ & Design loads \\
\hline 1 & 2682 & 73.2 & 1.793 & 17.918 & 59.202 & 68.37 & 2.848 & 92.259 \\
\hline 2 & 2359 & 1592 & 19.347 & 330.538 & 1139 & 1381 & 70.312 & 1821 \\
\hline 3 & 1877 & 1610 & 6.413 & 345.357 & 1184 & 1362 & 23.847 & 1837 \\
\hline 4 & 4027 & 2917 & 15.01 & 351.99 & 1633 & 1397 & 65.391 & 2179 \\
\hline 5 & 2603 & 1964 & 5.622 & 292.085 & 1657 & 1138 & 20.493 & 2031 \\
\hline 6 & 3603 & 2785 & 11.956 & \begin{tabular}{|l|}
377.904 \\
\end{tabular} & 1392 & 1501 & 77.134 & 2083 \\
\hline 7 & 1908 & 1631 & 6.505 & 337.438 & 1144 & 1350 & 24.382 & 1801 \\
\hline 8 & 1723 & 1043 & 13.162 & 215.751 & 722.16 & 910.249 & 112.92 & 1187 \\
\hline 9 & 1634 & 65.8 & 0.668 & 14.622 & 50.581 & 60.819 & 12.452 & \begin{tabular}{|l|l|}
81.404 & 0.668 \\
\end{tabular} \\
\hline 10 & 1013 & 743.5 & 2.807 & 162.017 & 561.145 & 601.711 & 10.458 & \begin{tabular}{|l|l|}
838.634 & 2.807 \\
\end{tabular} \\
\hline 11 & 1241 & 891.5 & 2.46 & 139.271 & 763.842 & 502.833 & 8.986 & \begin{tabular}{|l|l|}
925.083 & 2.46 \\
\end{tabular} \\
\hline 12 & 1006 & 781.4 & 2.837 & 148.991 & 504.969 & 666.873 & 10.647 & \begin{tabular}{|l|l|}
849.725 & 2.837 \\
\end{tabular} \\
\hline
\end{tabular}

\section{Conclusions}

The non-synchronization of pier excitations when calculating seismic loads is easily taken into account using existing software tools for the structure dynamic calculation. To do this, it is necessary to modify the calculation of the mode participant factor included in the formula for seismic loads and to calculate the set of seismic force matrices from the excitation of each of the supports separately.

Certain difficulties can be connected with the summation of modal forces and forces caused by pier excitations. If the vibration modes and pier excitations are statistically independent, the calculated force can be estimated as the root of the sum of the squares of its components. In more complicated cases, it is necessary to take into account the mode correlation and the correlation of pier excitations. If the assessment of the vibration mode correlation does not present any problems and is included in many software systems, the correlation coefficients of pier excitations still require additional research.

In the considered example, when the system is statically determinable and the mutual displacements of the supports do not cause additional forces in the system elements, taking into account the inhomogeneous acceleration field along the structure length results in a significant decrease of the design seismic loads. 


\section{References}

1. G.N. Kartsivadze Damage to road engineering structures during strong earthquakes. (M., Transport, 1969)

2. G.N. Kartsivadze Seismostability of road engineering structures (M., Transport, 1974)

3. G. S. Shestoperov, Seismostoikost mostov. (Moscow, Transport Publ., 1984)

4. A. M. Uzdin, I. O. Kuznetsova, Seismostoikost mostov (Saarbryuken (Germany), Palmarium Publ., 2014)

5. R.I. Skiner, W.H. Robinson, G.H. McVerry, An introduction to seismic isolation (New Zealand. John Wiley \& Sons.1993)

6. Y.P. Nazarov, N.A. Nikolaenko, Dynamics and Seismostability of structures. (M., Stroizdat, 1988)

7. E.V. Poznyak, O.V. Novikova, MPEI Vestnik, 5, 48-56 (2017). DOI: 10.24160/19936982-2017-5-48-56.

8. .P. Papadopoulos, A.G. Sextos, Soil Dynamics and Earthquake Engineering, 131, 106013 (2020) https://doi.org/10.1016/j.soildyn.2019.106013

9. L.N. Dmitrovskaya, A.M. Uzdin, First European Conference on Earthquake Engineering and Seismology, 32, (2006)

10. A. Der-Kiureghian Earthquake Engineering and Structural Dynamics. 9(5), 419-435 (1981).

11. A. Der-Kiureghian, Y. Nakamuro, Earthquake Engineering and Structural Dynamics, 22, 943-956 (1993)

12. A.K. Chopra Dynamics of structures: Theory and applications to earthquake engineering, (Prentice-Hall, Englewood Cliffs, NJ, 1995)

13. P.Yu. Semenov, V.A. Semenov Proceedings of the 5th International Conference on Computation of Shell\&Spatial Structures. (2005)

14. A.G. Tyapin Calculation of structures for seismic impacts, taking into account interaction with the ground base (Moscow: DIA Publ., 2013)

15. J.S. Przemieniecki Theory of matrix structural analysis. (Dover, 1985)

16. N.M. Newmark and E.Rosenblueth Fundamentals of earthquake engineering (PrenticeHall, Englewood Cliffs, N.J., 1971)

17. A.A. Petrov Proceedings of the 11th European conference on Earthquake Engineering (1998)

18. A. Perelmuter, V. Slivker Numerical Structural Analysis: Methods, Models and Pitfalls (Springer-Verlag Berlin Heidelberg GmbH, 2003). DOI 10.1007/978-3-540-36500-6

19. K. Katsuhisa. The proceedings of the 12-th World conference on earthquake engineering 0649 (2000)

20. S. Santa-Cruz, E. Heredia-Zavoni, R. Harichandran The proceedings of the 12-th World conference on earthquake engineering 0076 (2000)

21. O.P. Nesterova, N.A. Polorotova, A.M. Uzdin Structural Mechanics and Analysis of Constructions, 6, 40-44. 2019 